\title{
Les extrapreneurs : les entrepreneurs font le développement et les extrapreneurs, le sous-développement
}

\author{
Krasmer-Mobiank Kipoutou ${ }^{1}$ \\ Institut supérieur de gestion \\ Université Marien Ngouabi
}

\section{Introduction}

Le développement économique a pour agents les entrepreneurs; mais quel est l'agent du sousdéveloppement ? Cette question - apparemment sans intérêt - est lourde de conséquences. En effet, ne pas la poser revient à vouloir ne pas saisir le problème du sous-développement, du développement du sousdéveloppement, et donc du développement des pays sous-développés à sa base.

L'abandon du paradigme du développement pour celui de la lutte contre la pauvreté, l'échec des différentes décennies de développement décrétées par le PNUD, la désarticulation entre pays développés et pays sous-développés, les premiers devenant plus riches alors que les seconds s'appauvrissent de plus belle, les programmes de privatisation des entreprises publiques qui touchent les nombreux pays sous-développés et qui peuvent s'apparenter à une «recapitation» après la décapitation des indépendances, tout cela nous amène à nous demander s'il y a vraiment des entrepreneurs dans les pays considérés comme sous-développés ou économiquement en retard. Si Paul De Bruyne ne trouve dans ces pays que des commerçants et des artisans, et que les entrepreneurs qui s'occupent de l'activité industrielle sont absents ${ }^{2}$, nous nous interrogeons également si cette énumération est exhaustive et réaliste. Le caractère dépressif ou exothermique des systèmes économiques de ces pays nous amène à dire non.

Les propos d'André Piettre, que nous reprenons ciaprès, témoignent de la présence des agents qui, loin de faire le développement, nuisent à son processus :

Si tant d'efforts publics ou tant d'efforts privés, d'intention également généreuse, sont trop souvent restés inefficaces, la raison est qu'ils ne pénétraient pas jusqu'aux causes du mal, presqu'aux assises et jusqu'à l'esprit du régime. À côté d'investissements parfois contestables, des micro-réalisations ont soulevé d'ancestrales apathies. Car l'assistance ne peut tout faire. Et, il faut bien avouer que ces bénéficiaires n'ont pas toujours répondu à notre aide par d'égal effort. Des dissipations éhontées, des désordres sans nom et d'amères rancœurs excités par quelques ambitieux trop écoutés chez nous risquent de décourager les meilleures volontés. $^{3}$

Le caractère dépressif ou exothermique des systèmes économiques des pays sous-développés, la détérioration des termes de l'échange et les dévaluations des monnaies, la désarticulation entre le nord et le sud, les faillites et les privatisations des entreprises publiques au sud, entre autres, militent en faveur de la présence des agents qui, loin de faire du dévelop- 
pement, font du sous-développement. Ces agents, nous les appelons les extrapreneurs.

La théorie des entrepreneurs est une théorie du rôle, de la nature de leur fonction, de leurs origines, des conditions dans lesquelles ils fonctionnent le mieux. Nous ne reviendrons pas ici sur ces théories et renvoyons le lecteur redécouvrir les nombreux ouvrages spécialisés et d'économie qui en parlent. Par analogie, la théorie des extrapreneurs peut-être ébauchée en suivant le canevas de ces théories. L'objet de cet article est de faire part aux lecteurs des conclusions auxquelles nous sommes parvenu en étudiant les extrapreneurs, de tenter de sortir l'économie du développement dans cette sorte d'amnésie accommodante dans laquelle elle est cantonnée et de répondre à la question que nous nous sommes posée dès le départ.

\section{Les extrapreneurs aux antipodes des entrepreneurs}

L'extrapreneur est un agent qui, occupant la place ou la position de l'entrepreneur, favorise les externalisations pathologiques des ressources au profit de son milieu d'origine, de son réseau de référence ou les pratique pour son seul profit. Il ne dispose pas de base économique, n'a pas de compétences nécessaires, ou il est mis dans l'impossibilité de les éprouver, et ne peut donc pas par conséquent élargir la base de ses actions pour en faire la base du développement économique. Olivier Vallée ${ }^{4}$, qui donne une description parfaite de cet agent singulier, n'est pas allé jusqu'à lui trouver un autre nom. Il n'a pas échappé aux attitudes accommodantes en l'appelant entrepreneur accompagné du qualificatif africain, comme pour distinguer ces derniers des entrepreneurs occidentaux et pour dire qu'ils n'ont pas les mêmes pratiques, les mêmes méthodes et ne peuvent pas donner les mêmes résultats; quand même, il reconnaît que l'entrepreneur en Afrique est en devenir et que le phénomène et la réalité ne se sont pas encore rencontrés dans ce continent.

Le concept d'extrapreneur a été forgé à partir du préfixe extra et du mot entrepreneur pour coller à la réalité de cet agent singulier et tenir compte de ses logiques ou habitudes qui sont en dehors ou au-delà de celles des entrepreneurs dignes de ce nom. Au rang de ces logiques, nous retrouvons les logiques de notabilité, de quête de statut, de caste, de thésaurisation, de prestige, de promotion dans l'" establishment », de jouissance, de mercenaire du statu quo... qui sont étrangères aux logiques et stratégies de l'entreprise créatrice de richesses et facteur de développement. Toute vie, toute existence, dit André Piettre que nous citons ci -dessus, a un sens dans la double acceptation du terme. Elle a une signification: ainsi parle-t-on d'une vie de savant, d'ingénieur...; et elle a une direction, un déroulement dont on analyse les étapes. En nous basant sur le sens et la signification de l'existence des extrapreneurs, nous pouvons dire que ceux-ci s'identifient à la fin de la bourgeoisie occidentale. Ils commencent par la fin. Ils sont déjà sénescents alors qu'ils n'ont connu ni la pétulance, ni l'intrépidité, ni le volontarisme de la jeunesse et de l'adolescence. Le corollaire d'une telle situation de quête de statut ou de confinement à celui-ci, l'habitus d'innovation, de rationalité, d'amélioration de l'allocation des ressources, de l'observation d'une quelconque éthique sont absents.

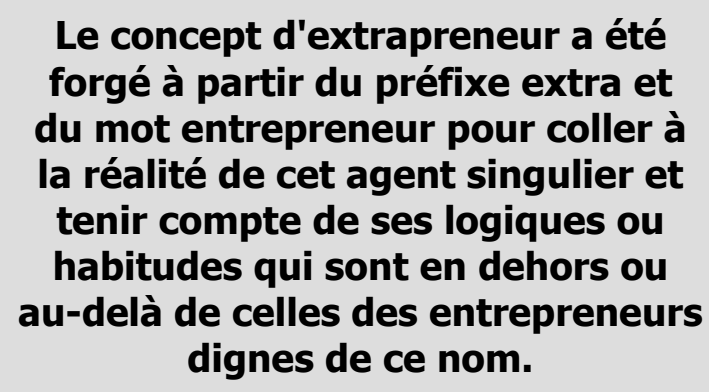
forgé à partir du préfixe extra et du mot entrepreneur pour coller à la réalité de cet agent singulier et tenir compte de ses logiques ou habitudes qui sont en dehors ou au-delà de celles des entrepreneurs dignes de ce nom.

La science économique connaît les entrepreneurs. Elle connaît également les repreneurs et les intrapreneurs dans le contexte de l'occident. Elle vient même de découvrir les non-entrepreneurs dont Hisrich et Peters nous présentent huit portraits types. Il s'agit: du bouillant, de la simple, de la prima donna, du blanc-bec, de la méticuleuse, du ringard, du fourvoyé et de l'inventeur ${ }^{5}$. Mais elle ne connaît pas les extrapreneurs que nous classons néanmoins parmi les non-entrepreneurs et qui peuvent être rapprochés des fourvoyés. Par une sorte de raccourci conceptuel accommodant, elle a eu tendance à assimiler les hommes et les femmes que l'on trouve à la tête de nombreuses structures des pays sous- 
développés de façon générale, des pays africains en particulier, d'entrepreneurs. Et on n'est pas étonné que ces contrées soient sous-développées et en sousdéveloppement; alors que, sous d'autres cieux, les entrepreneurs et les entreprises font le développement. Il faut donner raison à Jean-Paul Sartre, qui, en son temps, disait : " malnommer les choses, c'est ajouter aux malheurs du monde ».

L'ignorance des extrapreneurs est d'autant plus inadmissible que la science économique s'est depuis engagée sur l'analyse de l'hétérogénéité du milieu économique, non seulement en distinguant les unités selon leurs dimensions, mais encore en reconnaissant l'extrême diversité des sujets économiques. Cette diversité tient à une différence de rationalité, aux différences d'horizons économiques et de l'inégalité de leur force et de leur influence. En retenant par exemple le critère de rationalité, André Marchal classe les individus en ceux qui sont ultra-rationnels - qui agissent en fonction de la connaissance des lois économiques, de leur expérience des hommes et de l'intuition de l'avenir -; les individus infra-rationnels, qui sont conditionnés par le milieu, esclaves de la mentalité environnante, de la publicité et de la propagande; et enfin les homines oeconomicis, qui sont guidés par l'intérêt personnel et le principe de l'économie des forces ${ }^{6}$.

\section{Avec l'extrapreneur, l'entreprise et l'ensemble de l'économie ne sont que les expressions d'un mode d'administration approprié aux circonstances extérieures.}

L'observation de l'extrapreneur permet de dégager, à des degrés plus ou moins élevés, les caractéristiques suivantes : l'absence d'éthique ou le cynisme dont il fait preuve, son fort besoin de statut, d'affiliation, son faible besoin de réussite dans le cadre de l'entreprise, le laxisme dont il fait preuve et sa tendance à diluer le temps ou à fuir les responsabilités, le fait qu'il bénéficie de protections politiques, son vif intérêt pour les honneurs et pour les transactions sans contrepartie, et sa faible capacité de coordination d'où, par exemple, le fait que les conflits ne trouvent pas facilement de solutions en Afrique. Au sujet du laxisme, voici un autre exemple: si ce dernier ne prend pas de décision, ne tranche pas, tolère beaucoup de choses, c'est pour traduire la réalité de son pouvoir et le fait qu'il est prisonnier du groupe ou des pesanteurs sociologiques.

Les caractéristiques de l'extrapreneur agissent comme autant d'anti-valeurs qui, s'articulant à la chaîne de valeurs d'une entreprise, que ce soit aux activités commerciales, industrielles ou logistiques, annihilent ou inhibent toute possibilité de création de richesses et engagent ou amplifient le processus de dénaturation de l'entreprise et, partant, d'avortement du développement.

\section{À l'esprit d'entreprise, l'extrapreneur oppose l'esprit de son milieu d'origine ou de son réseau de référence. Son centre d'intérêt n'est pas l'entreprise.}

L'extrapreneur n'est pas animé par l'esprit d'entreprise. Les Africains seraient passés, après les indépendances, de l'esprit traditionnel à l'esprit bureaucratique, faisant l'impasse sur l'esprit d'entreprise. Ainsi, après l'esprit bureaucratique, sont-ils animés aujourd'hui par l'esprit de leur milieu d'origine ou de leur réseau de référence? D'où le fait qu'ils soient légion, les «entrepreneurs africains », mais installés dans leurs familles, leur administration publique, la politique et la guerre, les organisations internationales, les universités, les hôpitaux, les mosquées, les églises, les orchestres et les terrains vagues. D'où également le fait que leurs conduites possèdent des singularités encore affranchies d'un moule unique de l'intérêt et de la personnalité morale de l'entreprise. Ils sont animés par l'esprit d'extraprise ou par l'ensemble des qualités psychiques qui rendent impossible la réalisation heureuse d'une entreprise et qui ne permettent pas sa consolidation, sa reproduction à l'identique et son développement.

Par leur position, les extrapreneurs en profitent pour réaliser des transferts de ressources de façon pathologique de l'entreprise vers leurs centres d'intérêt ou pour les favoriser. La rapidité avec laquelle ils prennent le pouvoir n'a d'égale que celle avec laquelle ils se livrent aux pratiques d'externalisation pour leur enrichissement personnel ou les favoriser au profit de leur milieu d'origine ou de leur réseau. 
L'extrapreneur nous fait penser, par sa position et son comportement, au citoyen de la Grèce antique qui s'adonnait bien à un métier : artisanat, labeur des champs, travaux de port; qui participait s'il était riche à des placements financiers; qui faisait travailler les esclaves et plaçait son argent dans les mines; mais dont le véritable centre d'intérêt était ailleurs, en principe du moins. Son vrai centre d'intérêt était à l'agora, à l'ecclésia, au théâtre, au gymnase, aux concours et aux jeux. L'avantage que le citoyen de la Grèce antique a sur l'extrapreneur est que ce dernier avait des esclaves, des métèques ou des étrangers domiciliés et des affranchis pour s'occuper des choses économiques.

L'apparition des extrapreneurs est due à l'atrophie du statut de l'entrepreneur, à la dénaturation de leurs stratégies, au non-fonctionnement normal des filières d'accès à la fonction d'entrepreneur, à l'absence de liberté et de démocratie. Ils représentent les candidats entrepreneurs, c'est-à-dire les élites dynastiques, la classe moyenne, les intellectuels révolutionnaires, les administrateurs coloniaux et les leaders nationalistes - ou ceux qui avaient vocation à le devenir ou à l'être, mais dont les stratégies sont fourvoyées ou qui affichent des comportements déviants. Ils sont cooptés par les régimes en place, bénéficient d'une position de rente ou de quasi-rente et où, pour se maintenir, il leur faut reconnaître les dits régimes et leurs réseaux d'accès à la richesse. Ces régimes sont eux-mêmes impliqués dans la fuite des capitaux, l'exploitation des matières premières, comme ils l'étaient jadis dans l'extradition passée des hommes.

En Afrique, l'avènement des extrapreneurs locaux au pouvoir date des indépendances. En effet, à partir de cette période, ceux qui ont pris le pouvoir ont été promus du jour au lendemain à la suite du départ massif des populations expatriées. Ils se sont transformés en colons dans leur propre pays, marginalisant ou excluant la grande majorité des peuples à la prise de décisions et à l'administration de leur pays.

L'Africain n'est pas ontologiquement entrepreneur, dit Olivier Vallée cité ci-dessus. Cela ne procède pas de son milieu social ou de la pauvreté en capitaux de son milieu, mais du fait que l'homo économicus en Afrique a été remis en cause par la colonisation, par sa participation à la fois comme sujet et objet de l'esclavage; les dominations économiques, politiques et culturelles n'ont fait que reproduire le processus et le maintenir dans cette situation.

\section{Les structures à la tête desquelles se retrouvent les extrapreneurs présentent un profil de proie, ce sont des entreprises-choses.}

Les structures que les extrapreneurs créent, animent ou dirigent sont organisées de façon à privilégier les pressions extérieures jugées stratégiquement plus importantes : LES EXTRAPRISES. Les extraprises font de la redistribution ou subissent des externalisations pathologiques de ressources. Les plus efficaces d'entre elles consomment leurs capitaux. En Afrique, par exemple, l'entreprise est créée en réseau et les créateurs mettent en avant leurs responsabilités sociales, familiales ou politiques. La création de ce genre de structures est facile, mais leur maintien en vie et en fonctionnement à sa pleine capacité est par contre très difficile, parce qu'elles sont dépressives, victimes des externalisations pathologiques qui corrodent tout processus de développement ou de reproduction à l'identique. Octave Gelinier qualifie les sociétés sous-développées de sociétés sans entreprise, en réservant ce mot à l'unité qui a un pouvoir de décision autonome ${ }^{7}$. L'extraprise ne dispose pas des moyens d'une stratégie propre.

Le contexte dans lequel les extrapreneurs prolifèrent est celui de l'économie dépendante ou subordonnée. Par le manque de rationalité qui les caractérise, le cynisme dont ils font preuve, les pressions qu'ils subissent, les externalisations pathologiques des ressources ou richesses qu'ils favorisent ou pratiquent donnent aux entreprises un profil de proie, les transforment en machin formé d'un nombre donnée de contrats que l'on parasite.

L'extrapreneur détruit les ressources ou les richesses. C'est un agent de dénaturation et de désintégration de l'entreprise qui ne favorise pas la réallocation avantageuse des ressources au niveau du pays. Il fait 
de la destruction-destructrice des ressources, qui est à opposer à la destruction-créatrice des ressources que réalise l'entrepreneur.

\section{L'extrapreneur fait de la destruction- destructrice, qui est à opposer à la destruction-créatrice que réalise I'entrepreneur.}

Les pratiques d'externalisation pathologiques des ressources qui sont confondues le plus souvent à la mauvaise gestion, même quand elles bénéficient à leurs auteurs, font en fait partie ou relèvent d'un système d'administration favorable aux pressions extérieures, jugées stratégiquement plus importantes. Ce système tend à faire des clientèles, à transformer les opposants éventuels en partisans du pouvoir, à diriger par la corruption ou pour contenter les membres du réseau ou des milieux d'origine des extrapreneurs. Il est comparable au système des avantages financiers, des solidarités, des devoirs réciproques qui prévalaient en Europe féodale pour maintenir les seigneurs en nobles de cour dociles. Ces pratiques vont actuellement au-delà de la mission historique de courroie de transmission ou d'agent d'affaires que Fanon attribue aux classes dirigeantes des pays sous-développés, en participant activement à l'externalisation pathologique des ressources ou des richesses et en défendant par tous les moyens cette position ou le rôle d'interface ou d'intermédiation qui est le leur.

Les interventions des extrapreneurs reproduisent les fondements de la valeur dans les pays sousdéveloppés en général, et en Afrique en particulier : la rareté et la nature subordonnée de l'économie. Ces interventions sont à la base d'un enrichissement privé qui ne se dissémine pas dans la société. Leurs stratégies ou pratiques se calquent sur les projets, les investissements, les dépenses, les ventes ou les contrats de l'entreprise. Elles sont qualifiées également de récupération à la marge, entrainant la dérive par rapport aux systèmes de protection des équilibres monétaires, le non-respect des principes régissant le bon fonctionnement de l'entreprise. Elles donnent lieu aux mécanismes du type transaction sans contrepartie, dépenses onéreuses, surfacturation des achats, sous-factuaration des ventes, détournement des fonds ou des ressources, surpaiement des cadres de direction, etc. qui alimentent la fuite des capitaux et autres évasions fiscales.

L'extrapreneur peut s'entourer de toute une équipe de prétendus collaborateurs. Cette superstructure ne vise pas l'efficacité ou la réalisation des performances économiques, techniques ou commerciales... Ces considérations sont vite marginalisées, battues en brèche pour mettre en avant l'embauche des amis, des parents, des clientèles qui y trouvent une niche écologique pour couler des jours aisés à l'abri des tracasseries financières. Cette superstructure se présente soit sous forme de "népostructure » lorsque l'extrapreneur privilégie ses relations familiales et amicales pour constituer l'équipe de direction, soit sous forme de «ploutostructure » lorsque ce sont les apports en argent ou en capital qui déterminent la composition de l'équipe de direction. Les fonctions liées à ces niveaux restent dominées par des agents qui ne sont pas encore acquis à la nécessité de l'efficacité, de l'utilisation rationnelle des ressources et de la compétence.

Les extrapreneurs font partie des forces de corrosion du développement qui non seulement bloquent ou retardent le développement, mais qui continuent de peser lourdement sur le processus une fois qu'il est en train. Ils sont à la base du développement extraverti qui n'est pas développement, mais développement du sous-développement, selon l'expression bien connue de Samir Amin. Avec eux, les caractères spécifiques du sous-développement - inégalités internes croissantes, distorsion conséquente de l'allocation des ressources, marginalisation et dépendance, etc. - ne sont pas réduits, mais de plus en plus marqués

Avec les extrapreneurs, les entreprises et les États sont perméables, extravertis et leurs fonctionnements adaptés pour répondre aux sollicitations et exigences de l'extérieur. Avec leur présence, les politiques économiques de développement, de redressement et les plans sont voués à l'échec. Les pratiques qui transforment les entreprises en extraprises sont les mêmes qui mettent le pays sur la pente du sousdéveloppement et qui entraînent le développement du sous-développement, qui prend nécessairement 
l'allure de périodes de croissance rapide soudainement bloquées, de miracles sans lendemains, de décollages ratés.

Les sociétés sous-développées sont également des sociétés d'extrapreneurs. Les populations de ces sociétés sont réduites à rechercher des niches écologiques auxquelles elles articulent des logiques étrangères et à externaliser ainsi des ressources. Les pratiques d'externalisation des ressources ou des richesses se sont généralisées et banalisées au point où nous pouvons avancer que les sociétés sousdéveloppées ou en sous-développement sont également des sociétés d'extrapreneurs.

\section{L'extrapreneur constitue le prince des facteurs du sous-développement et du développement du sous-développement.}

\section{Conclusion}

Nous pouvons dire qu'avec les extrapreneurs, les facteurs de sous-développement ont trouvé leur prince. En effet, si de nombreux ouvrages et auteurs parlent du sous-développement, des facteurs de sousdéveloppement, de bourgeoisie non investisseuse et jouisseuse, ils ne vont pas jusqu'à dévoiler cette prétendue bourgeoisie dans son rôle de destruction- destructrice ni jusqu'à la mettre au centre du processus de sous-développement. En un mot, le prince des facteurs du sous-développement restait dans les coulisses, ou le toréador derrière la muleta.

\section{Notes et références}

1 Krasmer-Mobiank Kipoutou est docteur ès sciences économiques. Il est assistant à l'Université Marien Ngouabi du Congo-Brazzaville et enseignant permanent à l'Institut supérieur de gestion. En dehors des recherches qu'il mène sur les extrapreneurs, il s'intéresse également à l'entreprisation des activités économiques ou au processus de prise en main des activités économiques par les entreprises aussi bien dans le contexte de l'occident que dans celui des pays sous-développés.

2 De Bruyne, P. (1963). Esquisse d'une théorie de l'administration des entreprises, Paris, Dunod.

3 Piettre, A. (1964). Les trois âges de l'économie et de la civilisation occidentale, Paris, Fayard.

4 Vallée, O. (1992). Les entrepreneurs africains, Paris, Ed. Syros Alternatives du Seuil.

5 Hisrich, R.D. et M.P. Peters (1992). L'Entrepreneurship : lancer, élaborer et gérer une entreprise, Paris, Economica.

6 Marchal, A., cité par R. Barre (1955). Économie politique, I, Paris, PUF.

7 Gelinier, O. (1972). L'entreprise créatrice, Paris, Éditions Hommes et Techniques. 